\title{
BMJ
}

\section{Prophylactic antibiotics for burns patients: systematic review and meta-analysis}

\author{
Tomer Avni, resident, ${ }^{1}$ Ariela Levcovich, senior physician, , ${ }^{2}$ Dean D Ad-El, head of department, ${ }^{3}$ Leonard \\ Leibovici, head of department, ${ }^{1}$ Mical Paul, consultant ${ }^{4}$
}

${ }^{1}$ Department of Medicine E, Rabin Medical Center, Beilinson Hospital, Sackler Faculty of Medicine, TelAviv University, Israel

${ }^{2}$ Department of Medicine B, Rabin Medical Center, Beilinson Hospital

${ }^{3}$ Department of Plastic Surgery and Burns, Rabin Medical Center, Beilinson Hospital

${ }^{4}$ Unit of Infectious Diseases, Rabin Medical Center, Beilinson Hospital Correspondence to: M Paul paulm@post.tau.ac.il

Cite this as: $B M J$ 2010;340:c241 doi:10.1136/bmj.c241

\section{ABSTRACT}

Objective To assess the evidence for prophylactic treatment with systemic antibiotics in burns patients.

Design Systematic review and meta-analysis of randomised or quasi-randomised controlled trials recruiting burns inpatients that compared antibiotic prophylaxis (systemic, non-absorbable, or topical) with placebo or no treatment.

Data sources PubMed, Cochrane Library, LILACS, Embase, conference proceedings, and bibliographies. No language, date, or publication status restrictions were imposed.

Review methods Two reviewers independently extracted data. The primary outcome was all cause mortality. Risk or rate ratios with $95 \%$ confidence intervals were pooled with a fixed effect model if no heterogeneity was present. Results 17 trials were included. Trials that assessed systemic antibiotic prophylaxis given for 4-14 days after admission showed a significant reduction in all cause mortality (risk ratio $0.54,95 \%$ confidence interval 0.34 to 0.87 , five trials). The corresponding number needed to treat was 8 (5 to 33), with a control event rate of $26 \%$. Perioperative non-absorbable or topical antibiotics alone did not significantly affect mortality. There was a reduction in pneumonia with systemic prophylaxis and a reduction in wound infections with perioperative prophylaxis. Staphylococcus aureus infection or colonisation was reduced with anti-staphylococcal antibiotics. In three trials, resistance to the antibiotic used for prophylaxis significantly increased (rate ratio $2.84,1.38$ to 5.83 ). The overall methodological quality of the trials was poor.

Conclusions Prophylaxis with systemic antibiotics has a beneficial effect in burns patients, but the methodological quality of the data is weak. As such prophylaxis is currently not recommended for patients with severe burns other than perioperatively, there is a need for randomised controlled trials to assess its use.

\section{INTRODUCTION}

Severe burns are an important health burden worldwide and affect young healthy adults and children. ${ }^{12}$ Infections among burns patients are a major problem; the reported incidence of nosocomial infections varies at 63-240 per 100 patients and 53-93 per 1000 patient days, depending mainly on the definitions used. ${ }^{34}$ Infections are independently associated with adverse outcomes and mortality. ${ }^{34}$ In a series of 175 patients with severe burns, infections preceded multiorgan dysfunction in $83 \%$ of patients and were considered as the direct cause of death in 36\% of patients who died. ${ }^{5}$

In burns patients infections arise from multiple sources. Burn wounds become rapidly infected with Gram positive bacteria, mainly staphylococci, that are normal deep inhabitants of the sweat glands and hair follicles exposed by the burn. ${ }^{6}$ The moist, vascular burn eschar further fosters microbial growth. Gram negative bacterial infections result from translocation from the colon because of reduced mesenteric blood flow at the time of burn and subsequent insults. ${ }^{7}$ Furthermore, several immune deficits have been described among burns patients, including impaired cytotoxic T lymphocyte response, myeloid maturation arrest causing neutropenia, impaired neutrophil function, and decreased macrophage production. ${ }^{68-10}$ Finally, burns patients can incur hospital acquired infections common to other patients in intensive care units, including intravascular catheter related infections and ventilator associated pneumonia, with an overall incidence of infection higher than that of other patients in intensive care units. ${ }^{34}$

Antibiotic prophylaxis reduces mortality, bacteraemia, and ventilator associated pneumonia among patients in intensive care units. ${ }^{11}{ }^{12}$ Similarities between intensive care and burns patients suggest possibly similar benefit of prophylaxis. Both populations are critically ill, and bacterial translocation from the colon is an important source of infection, as are foreign bodies and invasive procedures. In burns patients the skin is an additional source of infection, and they have a higher degree of immunosuppression. Nevertheless, there is a broad and uniform consensus in the current literature that prophylaxis with systemic antibiotics should not be given to patients with severe burns. Recommendations for management do not address systemic antibiotic prophylaxis ${ }^{13}$ or explicitly state that prophylactic antibiotics are not recommended. ${ }^{14-18}$ The rationale given is lack of evidence, no benefit, or risk for adverse events, mainly colitis associated with Clostridium difficile and induction of antibiotic 


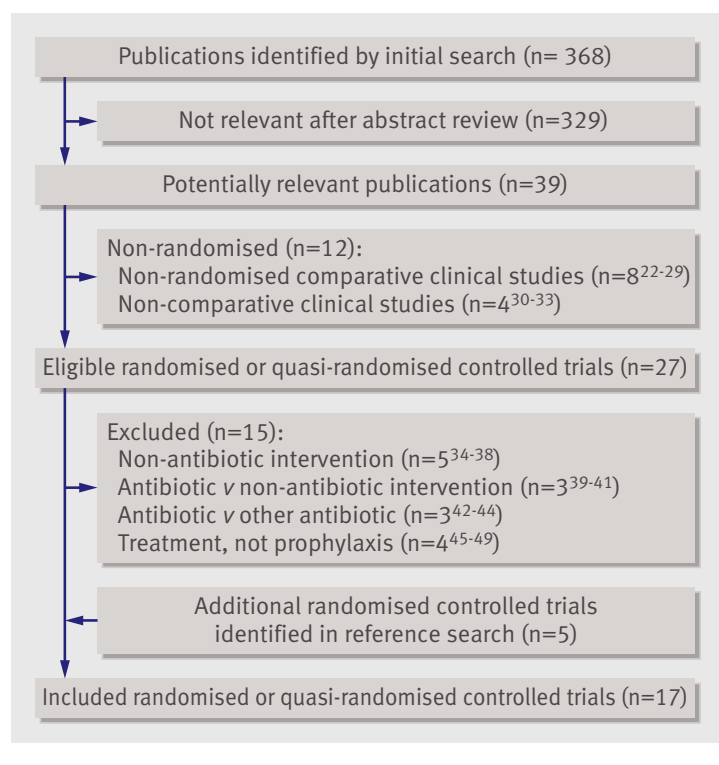

Fig 1| Identification of studies for inclusion

resistance. Indeed, most episodes of bloodstream infection after the first week are caused by hospitaltype multidrug resistant bacteria. ${ }^{419}$ Recommendations regarding perioperative prophylaxis vary and most sources recommend limited perioperative prophylaxis only for those with severe burns $>40 \%$ total body surface area). ${ }^{141617}$

We performed a systematic review and meta-analysis of randomised and quasi-randomised controlled trials assessing antibiotic prophylaxis for burns patients, both in the perioperative and general setting. We primarily examined the effect of prophylaxis on all cause mortality.

\section{METHODS}

\section{Selection criteria}

We included randomised controlled trials or quasi-randomised trials (with inadequate allocation generation methods), recruiting inpatients with burns injuries (any total body surface area or burn degree, with or without inhalation injury), regardless of publication status or language. The intervention assessed was antibiotic prophylaxis versus placebo or no treatment. Prophylaxis was defined as antibiotics administered to patients without documented infection regardless of systemic inflammatory signs, including systemic antibiotics given intravenously, orally, or intramuscularly; nonabsorbable oral antibiotics; or topical (wound dressing or inhalation) antibiotics. Regimens including both systemic and non-absorbable or topical antibiotics were included in the systemic category. Antibiotics could be administered at any time after admission ("general") or specifically targeted at a surgical procedure ("perioperative"). We excluded topical non-antibiotic antimicrobial ointments or dressings (silver with or without sulpha, iodine, or mafenide) and antifungals, unless applied identically to intervention and control arms. We excluded dose or schedule comparisons of the same antibiotics.

\section{Outcomes}

The protocol defined primary outcome was all cause mortality 100 days after randomisation. None of the studies reported 100 day data or similar, nor at another fixed point in time, and so we extracted in hospital mortality from all the studies, per protocol. Secondary outcomes included bacteraemia, pneumonia (including ventilator associated pneumonia), infection of the burn wound, length of stay in hospital, infections caused by Pseudomonas aeruginosa, Staphylococcus aureus, and meticillin resistant $S$ aureus (MRSA), resistance induction, fungal infections (fungaemia or other clinical fungal infection), and adverse events. Resistance induction was defined per protocol as clinical infection (not colonisation) caused by bacteria resistant to one or more of the antibiotics included in the prophylactic regimen. Studies, however, reported only on selected "resistant isolates" (including both clinical and colonising bacteria); these data and their definitions were extracted. Similarly, we accepted and documented other outcomes definitions used in individual studies.

\section{Search methods}

We searched PubMed (1966 to February 2009), Cochrane Library (issue 4, 2008), LILACS (1982 to February 2009), Embase (1974 to October 2009), and conference proceedings (Interscience Conference on Antimicrobial Agents and Chemotherapy 1995-2008; European Congress of Clinical Microbiology and Infectious Diseases 2000-8; Annual Meeting of the American Burn Association 2001-9; Congress of the International Society for Burn Injuries 2007; and the Annual Southern Region Burn Conference 2008-9). We crossed the words "burn" or "total body surface area or TBSA" and their MESH terms with the terms "antibiotic," "infection", "sepsis", or "bacteremia". For PubMed, this was combined with the Cochrane highly sensitive filter for randomised controlled trials. ${ }^{20} \mathrm{We}$ scanned the references of all included articles for additional studies. Authors were contacted to complement data on mortality and trial methods (one author ${ }^{21}$ supplied additional data on methods).

\section{Data collection}

Two reviewers (TA and AL) independently inspected each reference identified by the search, scanned ful texts of relevant studies, applied the inclusion criteria, and extracted the data. Disagreements on data extraction were resolved by discussion with a third reviewer (MP). We assessed risk of bias in duplicate using domain based evaluation, classifying studies primarily according to the risk of non-random allocation of patients to the intervention arm (sequence generation) and concealment of this process (allocation concealment). These were graded as adequate, unclear, or not described and inadequate (for example, alternation, allocation by day of admission, hospital room), as recommended in the Cochrane Handbook. ${ }^{20}$ We also assessed blinding and intention to treat analysis. The effect of allocation concealment on results was assessed 
Table 1|Study characteristics of trials examining prophylactic antibiotics for burns patients in general settings. Figures are means (SD or SE) or median (range) unless stated otherwise

\begin{tabular}{|c|c|c|c|c|c|c|}
\hline Study and intervention details & $\begin{array}{l}\text { Intervention } \\
\text { duration (days) }\end{array}$ & $\begin{array}{l}\text { No of patients } \\
\text { randomised }\end{array}$ & $\begin{array}{c}\text { Age } \\
\text { (years) }\end{array}$ & $\begin{array}{l}\text { TBSA } \\
(\%)\end{array}$ & $\begin{array}{l}\text { 3rd degree } \\
\text { burns }(\%)\end{array}$ & $\begin{array}{l}\text { Inhalation } \\
\text { injury (\%) }\end{array}$ \\
\hline \multicolumn{7}{|l|}{ Barret $2001^{52}$} \\
\hline $\begin{array}{l}\text { Non-absorbable per nasogastric tube polymyxin } \mathrm{E} \text {, } \\
\text { tobramycin, amphotericin B }\end{array}$ & \multirow{2}{*}{$\begin{array}{l}\text { Until open burn area } \\
\qquad 10 \% \text { TBSA }\end{array}$} & 11 & $8(1)$ & $67(6)$ & 100 & 75 \\
\hline Placebo & & 12 & $9.4(2)$ & $58(6)$ & 100 & 63.6 \\
\hline \multicolumn{7}{|l|}{ De la Cal $2005^{53}$} \\
\hline $\begin{array}{l}\text { Systemic intravenous cefotaxime + oropharyngeal } \\
\text { paste and non-absorbable digestive administration } \\
\text { of polymyxin E, tobramycin, amphotericin B }\end{array}$ & 4 & 58 & $41.4(17.1)$ & $34(21.4)$ & $19.3(15.3)$ & 64.2 \\
\hline Placebo & & 59 & $48.2(28.5)$ & $37.7(21.1)$ & $19.0(18.8)$ & 68.5 \\
\hline \multicolumn{7}{|l|}{ Desai $1991^{54}$} \\
\hline Topical gentamicin $1 \%$ cream & \multirow{2}{*}{ Until wound healing } & 7 & $11.4(1.2)$ & $35(7)$ & $20(9)$ & \multirow{2}{*}{ NS } \\
\hline No treatment & & 8 & $9.5(1.6)$ & $50(6)$ & $32(7)$ & \\
\hline \multicolumn{7}{|l|}{ Deutsch $1990^{55}$} \\
\hline $\begin{array}{l}\text { Systemic and non-absorbable oral/nasogastric tube } \\
\text { erythromycin, neomycin, nystatin }\end{array}$ & \multirow[t]{2}{*}{10} & 15 & $44.7(15-79)$ & $49.9(22-91)$ & $26.3(0-75)$ & 26.7 \\
\hline No treatment & & 12 & $35(18-75)$ & $44.9(20-75)$ & $26.3(0-50)$ & 41.7 \\
\hline \multicolumn{7}{|l|}{ Durtschi $1982^{56}$} \\
\hline Systemic intravenous or oral penicillin & \multirow{2}{*}{5} & 25 & $31.1(18-77)$ & $14.9(1-70)$ & \multirow{2}{*}{ NS } & \multirow{2}{*}{ NS } \\
\hline Placebo & & 26 & $36.8(18-66)$ & $20(1-91)$ & & \\
\hline \multicolumn{7}{|l|}{ Kimura $1998^{57}$} \\
\hline $\begin{array}{l}\text { Systemic per nasogastric tube sulfamethoxazole- } \\
\text { trimethoprim }\end{array}$ & \multirow[t]{2}{*}{10} & 21 & $44(10-91)$ & $49(22-87)$ & \multirow[t]{2}{*}{ NS } & 52 \\
\hline Placebo & & 19 & $48(12-85)$ & $43(20-80)$ & & 63 \\
\hline \multicolumn{7}{|l|}{ Levine $1978^{65}$} \\
\hline Inhalation gentamicin & \multirow{2}{*}{10} & 12 & 28.1 & 53.8 & \multirow{2}{*}{ NS } & 100 \\
\hline Placebo & & 18 & 34.3 & 57.6 & & 100 \\
\hline \multicolumn{7}{|l|}{ Livingston $1990^{21}$} \\
\hline Topical neomycin and bacitracin & \multirow{2}{*}{ Until graft healing } & 18 & $\begin{array}{l}20 \% \text { TBSA } 46(22) ; 20-40 \% \\
27(5) ;>40 \% 49(10)\end{array}$ & $\begin{array}{l}<20 \% \text { TBSA } 14 \text { (5); } 20-40 \% \\
29(7) ;>40 \% 47(6)\end{array}$ & NS & 22.2 \\
\hline Normal saline & & 15 & $\begin{array}{l}20 \% \text { TBSA } 43(27) ; 20-40 \% \\
34(20) ;>40 \% 43(19)\end{array}$ & $\begin{array}{l}<20 \% \text { TBSA } 11(3) ; 20-40 \% \\
28(6) ;>40 \% 53(16)\end{array}$ & NS & 33.3 \\
\hline \multicolumn{7}{|l|}{ Lowbury $1968^{58}$} \\
\hline Topical silver nitrate + gentamicin & \multirow{2}{*}{$\begin{array}{l}\text { Until burns had healed } \\
\quad \text { or were grafted }\end{array}$} & 21 & \multirow{2}{*}{ NS } & \multirow{2}{*}{$\ll 30$} & \multirow{2}{*}{ NS } & \multirow{2}{*}{ NS } \\
\hline Topical silver nitrate & & 20 & & & & \\
\hline \multicolumn{7}{|l|}{ Munster $1989^{59}$} \\
\hline Systemic intravenous polymyxin $B^{*}$ & \multirow{2}{*}{7} & 22 & 34.4 & 29.3 & NC & NC \\
\hline No treatment & & 23 & 38.8 & 32.8 & INS & NS \\
\hline Ugburo $2004^{64}$ & & & & & & \\
\hline Systemic oral ampicillin + oxacillin & & 21 & $22.9(4.1)$ & $41.5(5.8)$ & & \\
\hline Systemic intravenous gentamicin+ oral erythromycin & 14 & 20 & $24.9(3.3)$ & $46(5.6)$ & NS & 0 \\
\hline None & & 20 & $23.3(3)$ & $44.3(6.3)$ & & \\
\hline
\end{tabular}

TBSA=total body surface area; MRSA=meticillin resistant $S$ aureus.

**Two sequential parts randomising patients to general systemic prophylaxis (first part) and perioperative systemic prophylaxis (second part), kept separate in our analyses.

through sensitivity analysis, with restriction of the analysis to studies with adequate allocation concealment.

Data analysis

Dichotomous outcomes (mortality, resistance development, and adverse events) are expressed per patient and count data (infections, bacteraemia) are given per patient day. Individual study results are expressed as risk ratios or rate ratios, respectively, with 95\% confidence intervals. Rate ratios were calculated as the ratio of events per patient day. Results were pooled with the Mantel-Haenszel fixed effect model (Review Manager (RevMan), version 5 for Windows, Cochrane
Collaboration, Oxford). We used $\chi^{2}$ test to determine heterogeneity $(\mathrm{P}<0.1)$ or an $\mathrm{I}^{2}$ measure for inconsistency $(>50 \%) .{ }^{20}$ Outcomes with significant heterogeneity were not pooled. We anticipated heterogeneity related to total body surface area and degree of burn but did not perform subgroup analyses because of paucity of trials. Analyses were stratified by antibiotic mode and intervention: systemic antibiotics (which could be administered in the general or perioperative setting), non-absorbable antibiotics, and topical antibiotics. Because of paucity of trials in each analysis, we did not use any formal method to investigate publication bias. 
Table 2 Study characteristics of trials examining systemic prophylactic antibiotics for burns patients in perioperative settings. Figures are means (SD or SE) or median (range) unless stated otherwise

\begin{tabular}{|c|c|c|c|c|c|c|}
\hline Study and intervention details & $\begin{array}{c}\text { Intervention } \\
\text { duration (days) }\end{array}$ & $\begin{array}{l}\text { No of patients } \\
\text { randomised }\end{array}$ & $\begin{array}{c}\text { Age } \\
\text { (years) }\end{array}$ & $\begin{array}{c}\text { TBSA } \\
(\%)\end{array}$ & $\begin{array}{c}\text { Third degree } \\
\text { burns (\%) }\end{array}$ & $\begin{array}{c}\text { Inhalation } \\
\text { injury (\%) }\end{array}$ \\
\hline \multicolumn{7}{|l|}{ Alexander $1982^{51}$} \\
\hline Systemic intravenous cephalothin & \multirow{2}{*}{1} & 127 & $10.5(0.4)$ & \multirow{2}{*}{ NS } & \multirow{2}{*}{ NS } & \multirow{2}{*}{ NS } \\
\hline Placebo & & 122 & $10.8(0.4)$ & & & \\
\hline \multicolumn{7}{|l|}{ Alexander $1984^{50}$} \\
\hline Systemic antibiotics tailored to wound & \multirow{3}{*}{1} & 35 & \multirow{3}{*}{ NS } & $45.7 \%>30 \%$ & \multirow{3}{*}{ NS } & \multirow{3}{*}{ NS } \\
\hline cultures & & & & & & \\
\hline No treatment & & 34 & & $38.2 \%>30 \%$ & & \\
\hline \multicolumn{7}{|l|}{ Munster $1989^{59}$} \\
\hline Systemic intravenous polymyxin B* & \multirow{2}{*}{5} & 6 & 47.7 & 54.8 & \multirow{2}{*}{ NS } & \multirow{2}{*}{ NS } \\
\hline No treatment & & 11 & 40.6 & 38.4 & & \\
\hline \multicolumn{7}{|l|}{ Piel $1985^{60}$} \\
\hline Systemic intravenous cephalosporin & \multirow[b]{2}{*}{1} & 25 & 33.6 & 42.6 & \multirow{2}{*}{ NS } & \multirow{2}{*}{ NS } \\
\hline Placebo & & 26 & 39.2 & 37 & & \\
\hline \multicolumn{7}{|l|}{ Ramos $2008^{61}$} \\
\hline Systemic intravenous cephalothin or & \multirow{3}{*}{2} & 46 & $39(21.7)$ & $21.6(21)$ & \multirow{3}{*}{ NS } & \multirow{3}{*}{ NS } \\
\hline antibiotics tailored to surveillance cultures & & & & & & \\
\hline No treatment & & 44 & $35(22)$ & $27.7(22.3)$ & & \\
\hline \multicolumn{7}{|l|}{ Rodgers $1997^{62}$} \\
\hline Systemic intravenous cefazolin & \multirow{2}{*}{1} & 10 & 1.5 & 10 & \multirow{2}{*}{ NS } & \multirow{2}{*}{ NS } \\
\hline Placebo & & 10 & 1.9 & 11 & & \\
\hline \multicolumn{7}{|l|}{ Steer $1997^{63} \dagger$} \\
\hline Systemic intravenous teicoplanin & \multirow{2}{*}{1} & 67 (110 episodes) & $38(23-54)$ & $8.5(4-18)$ & 44 & \multirow{2}{*}{ NS } \\
\hline Placebo & & 67 (110 episodes) & $42.5(26-56)$ & $8.0(4-16)$ & 45 & \\
\hline $\begin{array}{l}\text { TBSA=total body surface area; NS=not statec } \\
\text { *Two sequential parts randomising patients } \\
\text { separate in our analyses. } \\
\text { †Trial included patients before surgery }(46 \%\end{array}$ & $\begin{array}{l}\text { RSA=meticillin resi } \\
\text { eneral systemic pr } \\
\text { pisodes) or chang }\end{array}$ & $\begin{array}{l}\text { tant } S \text { aureus. } \\
\text { phylaxis (first part) an } \\
\text { of dressing. }\end{array}$ & d perioperative & systemic pro & yylaxis (secono & rt), kept \\
\hline
\end{tabular}

\section{RESULTS}

The search yielded 368 different publications, of which 39 were potentially relevant. Twenty seven studies were excluded (fig 1). We identified five trials through reference searching and altogether included 17 studies (37 trial arms), one of which was published as an abstract. ${ }^{2150-65}$ The trials were published from 1968 to 2008 and recruited 1113 patients (median 51, range 15149). Four trials recruited children ${ }^{51525462}$ and the others young adults (tables 1 and 2). The mean total body surface area affected was $>20 \%$ in 12 trials $(>30 \%$ in nine). Most trials did not report the number of patients with full thickness burns. Twelve administered systemic antibiotic prophylaxis; six trial arms assessed general ${ }^{5355-575964}$ and six perioperative prophylaxis..$^{5059-63}$ Systemic antibiotics usually targeted Gram positive bacteria and were given for a median of 8.5 days (range 4-14) in the general setting. Two trials assessing systemic general prophylaxis and none of the trials in the perioperative setting included a non-absorbable component. One trial assessed only non-absorbable prophylaxis, ${ }^{52}$ three trials topical antibiotic treatment, ${ }^{215458}$ and one trial inhalation antibiotics, ${ }^{65}$ targeting mainly Gram negative bacteria.

Five and six trials, respectively, described adequate sequence generation and allocation concealment. Both were inadequate in three quasi-randomised trials that used alternation or hospital number for sequence generation $^{55589}$ and were not described in all other trials (table 3 and fig 2). Patients and carers were blinded in seven trials (six assessing systemic prophylaxis). Results by intention to treat were reported in all but two trials.

\section{Primary outcome}

Nine trials reported all cause, in hospital mortality (fig 3). Systemic prophylaxis in the general setting was associated with a significant reduction in all cause mortality (risk ratio $0.54,95 \%$ confidence interval 0.34 to 0.87 , five trials, 272 patients), without significant het-

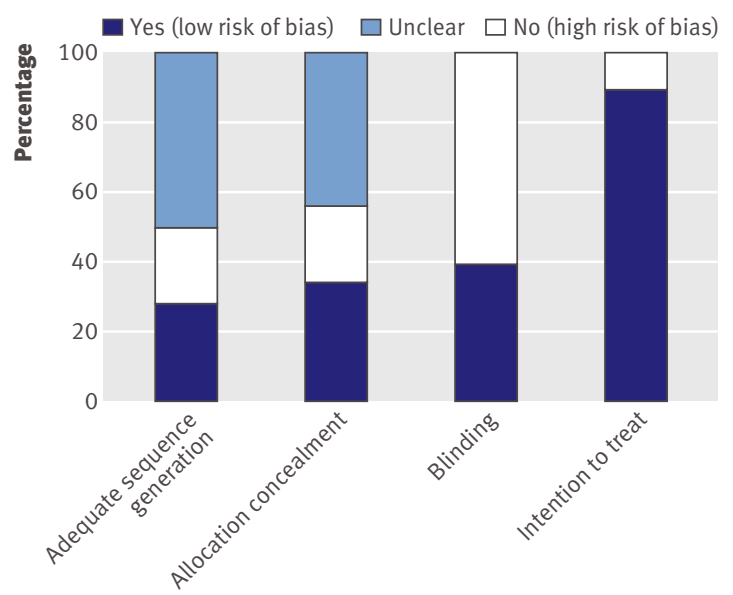

Fig 2 | Assessment of overall risk of bias 
Table $3 \mid$ Methods and outcome definitions used in individual trials

\begin{tabular}{|c|c|c|c|c|c|c|}
\hline Study & $\begin{array}{l}\text { Allocation } \\
\text { generation }\end{array}$ & $\begin{array}{l}\text { Allocation } \\
\text { concealment }\end{array}$ & Blinding* & $\begin{array}{l}\text { Intention to } \\
\text { treat analysis }\end{array}$ & $\begin{array}{c}\text { Secondary outcomes evaluated } \\
\text { and definitions }\end{array}$ & $\begin{array}{l}\text { Resistance development/ } \\
\text { surveillance cultures } †\end{array}$ \\
\hline Alexander $1982^{51}$ & $\begin{array}{l}\text { Adequate (random } \\
\text { choice of envelopes) }\end{array}$ & $\begin{array}{l}\text { Adequate (central } \\
\text { pharmacist) }\end{array}$ & $\begin{array}{l}\text { DB (placebo used, only } \\
\text { pharmacist aware of } \\
\text { treatment assignment) }\end{array}$ & Yes & $\begin{array}{l}\text { Wound infection (discharge of pus from } \\
\text { graft associated with graft loss); } P \\
\text { aeruginosa and } S \text { aureus infections; } \\
\text { hospital stay }\end{array}$ & NA. No surveillance \\
\hline Alexander $1984^{50}$ & Unclear (not stated) & Unclear (not stated) & $\begin{array}{l}\text { Open (treatment and } \\
\text { control patients placed in } \\
\text { different wards) }\end{array}$ & Yes & Bacteraemia; wound infection & $\begin{array}{l}\text { NA. Wound and blood } \\
\text { surveillance }\end{array}$ \\
\hline Barret $2001^{52}$ & $\begin{array}{l}\text { Adequate (random } \\
\text { number chart) }\end{array}$ & $\begin{array}{l}\text { Adequate (central } \\
\text { pharmacy) }\end{array}$ & $\begin{array}{l}\text { DB (placebo used, only } \\
\text { pharmacist aware of } \\
\text { treatment assignment) }\end{array}$ & Yes & $\begin{array}{l}\text { Pneumonia (by CDC criteria or similar }{ }^{66} \text { ); } \\
\text { systemic fungal infection; hospital stay }\end{array}$ & $\begin{array}{l}\text { NA. Wound, sputum, urine, } \\
\text { blood, gastric aspirates, } \\
\text { and stool surveillance }\end{array}$ \\
\hline De la Cal $2005^{53}$ & Unclear (not stated) & $\begin{array}{l}\text { Adequate (central, } \\
\text { pharmacy and kept in } \\
\text { sealed envelopes) }\end{array}$ & $\begin{array}{l}\text { DB (placebo used, only } \\
\text { pharmacist aware of } \\
\text { treatment assignment) + } \\
\text { evaluator blinded }\end{array}$ & No & $\begin{array}{l}\text { Pneumonia and bacteraemia (CDC } \\
\text { criteria }^{66} \text { ); wound infection (according to } \\
\text { previously proposed criteria }{ }^{67} \text { ); } \\
\text { candidaemia; } P \text { aeruginosa and } S \text { aureus } \\
\text { infections; hospital stay }\end{array}$ & $\begin{array}{l}\text { Unrelated; ventilator } \\
\text { associated pneumonia or } \\
\text { bacteraemia caused by } \\
\text { MRSA. Wound, throat, } \\
\text { rectal surveillance }\end{array}$ \\
\hline Desai $1991^{54}$ & Unclear (not stated) & Unclear (not stated) & Open & Yes & $\begin{array}{l}\text { Wound infection (chondritis, defined); } \\
\text { hospital stay }\end{array}$ & $\begin{array}{l}\text { Related; chondritis caused } \\
\text { by gentamicin-resistant } \\
\text { bacteria. Wound } \\
\text { surveillance }\end{array}$ \\
\hline Deutch $1990^{55}$ & $\begin{array}{l}\text { Inadequate } \\
\text { (chronological } \\
\text { alternation) }\end{array}$ & $\begin{array}{l}\text { Inadequate } \\
\text { (chronological } \\
\text { alternation) }\end{array}$ & Open & $\begin{array}{l}\text { Yes for mortality; } \\
\text { no for secondary } \\
\text { outcomes }\end{array}$ & $\begin{array}{l}\text { Wound and fungal infection (positive } \\
\text { wound cultures); bacteraemia; } P \\
\text { aeruginosa and } S \text { aureus infections; } \\
\text { hospital stay }\end{array}$ & NA. Wound surveillance \\
\hline Durtschi $1982^{56}$ & Unclear (not stated) & Unclear (not stated) & DB (placebo used) & Yes & $\begin{array}{l}\text { Wound infection (sepsis and warm, } \\
\text { spreading, painful cutaneous erythema); } \\
\text { bacteraemia; P aeruginosa, S aureus, and } \\
\text { fungal infections; hospital stay }\end{array}$ & $\begin{array}{l}\text { Unrelated; infections } \\
\text { caused by gentamicin- } \\
\text { resistant bacteria. Wound, } \\
\text { rectal surveillance }\end{array}$ \\
\hline Kimura $1998^{57}$ & Unclear (not stated) & $\begin{array}{l}\text { Adequate (central } \\
\text { pharmacy) }\end{array}$ & DB & Yes & $\begin{array}{l}\text { Pneumonia (by } C D C \text { criteria }^{66} \text { ); } P \\
\text { aeruginosa and } S \text { aureus infections }\end{array}$ & $\begin{array}{l}\text { Unrelated; MRSA } \\
\text { infections. No surveillance }\end{array}$ \\
\hline Levine $1978^{65}$ & Unclear (not stated) & Unclear (not stated) & $\begin{array}{l}\text { Single or DB (placebo } \\
\text { inhalations used) }\end{array}$ & Yes & $\begin{array}{l}\text { Pneumonia (pulmonary infiltrate); } \\
\text { bacteraemia; } P \text { aeruginosa infections }\end{array}$ & NA. Blood surveillance \\
\hline Livingston $1990^{21}$ & $\begin{array}{l}\text { Adequate (cards } \\
\text { shuffled at } \\
\text { assignment) }\end{array}$ & $\begin{array}{l}\text { Adequate (cards } \\
\text { placed in sealed } \\
\text { envelopes) }\end{array}$ & Open & Yes & $\begin{array}{l}\text { Wound infection ( }>10 \% \text { graft loss and }>10^{5} \\
\text { organisms/g tissue, both in non-adherent } \\
\text { graft and recipient site; candida wound } \\
\text { infections; hospital stay }\end{array}$ & $\begin{array}{l}\text { Unrelated; MRSA } \\
\text { infections. Wound } \\
\text { surveillance }\end{array}$ \\
\hline Lowbury $1968^{58}$ & $\begin{array}{l}\text { Inadequate } \\
\text { (alternation) }\end{array}$ & $\begin{array}{l}\text { Inadequate } \\
\text { (alternation) }\end{array}$ & $\begin{array}{l}\text { Open (no placebo, no } \\
\text { blinding described) }\end{array}$ & Yes & $P$ aeruginos $a$ and $S$ aureus infections & $\begin{array}{l}\text { Related; infections caused } \\
\text { by gentamicin-resistant } \\
\text { bacteria. Wound } \\
\text { surveillance }\end{array}$ \\
\hline Munster $1989^{59}$ & $\begin{array}{l}\text { Inadequate } \\
\text { (randomised by } \\
\text { hospital number) }\end{array}$ & $\begin{array}{l}\text { Inadequate } \\
\text { (randomised by } \\
\text { hospital number) }\end{array}$ & $\begin{array}{l}\text { Open (no placebo, no } \\
\text { blinding described) }\end{array}$ & Yes & No secondary outcome & NA. No surveillance \\
\hline Piel $1985^{60}$ & Unclear (not stated) & Unclear (not stated) & $\begin{array}{l}\text { Open (intervention listed on } \\
\text { bedside flow chart) }\end{array}$ & Yes & Bacteraemia & $\begin{array}{l}\text { NA. Wound and blood } \\
\text { surveillance }\end{array}$ \\
\hline Ramos $2008^{61}$ & Unclear (not stated) & $\begin{array}{l}\text { Adequate (sealed } \\
\text { envelopes) }\end{array}$ & Evaluator & Yes & $\begin{array}{l}\text { Wound infection (graft loss with swelling, } \\
\text { erythema, increased temperature, } \\
\text { tenderness or purulent discharge) }\end{array}$ & NA. Wound surveillance \\
\hline Rodgers $1997^{62}$ & $\begin{array}{l}\text { Adequate (table of } \\
\text { random numbers) }\end{array}$ & Unclear (not stated) & $\begin{array}{l}\text { DB (placebo used, only } \\
\text { pharmacist and one un- } \\
\text { blinded investigator in the } \\
\text { operating room aware of } \\
\text { treatment assignment) + } \\
\text { evaluator }\end{array}$ & Yes & $\begin{array}{l}\text { Wound infection (clinical indication of } \\
\text { infection with positive quantitative skin, } \\
\text { wound biopsy, or blood cultures); } \\
\text { candida wound infection; bacteraemia; } P \\
\text { aeruginosa and } S \text { aureus infections }\end{array}$ & $\begin{array}{l}\text { NA. Wound and blood } \\
\text { surveillance }\end{array}$ \\
\hline Steer $1997^{63}$ & Unclear (not stated) & Unclear (not stated) & $\begin{array}{l}\text { DB (placebo used, } \\
\text { teicoplanin colour masked, } \\
\text { only pharmacist aware of } \\
\text { treatment assignment) }\end{array}$ & $\begin{array}{l}\text { Yes, but analysis } \\
\text { based on } \\
\text { episodes }\end{array}$ & $\begin{array}{l}\text { Wound infection (biopsy and quantitative } \\
\text { tissue or skin cultures); pneumonia } \\
\text { (respiratory infection manifested by } \\
\text { sepsis and increase of purulent } \\
\text { tracheobronchial secretions or worsening } \\
\text { pulmonary gas exchange); P aeruginosa } \\
\text { and S aureus infections; bacteraemia; } \\
\text { candidaemia }\end{array}$ & $\begin{array}{l}\text { Related and unrelated; } \\
\text { infections caused by } \\
\text { teicoplanin-resistant } \\
\text { staphylococci and MRSA. } \\
\text { Wound and blood } \\
\text { surveillance }\end{array}$ \\
\hline Ugburo $2004^{64}$ & $\begin{array}{l}\text { Adequate (table of } \\
\text { random numbers) }\end{array}$ & Unclear (not stated) & Open & Yes & $\begin{array}{l}\text { Wound infection (clinical infection using } \\
\text { previously proposed criteria }{ }^{68} \text {, with } \\
\text { histological and microbiological } \\
\text { confirmation); P aeruginosa and } S \text { aureus } \\
\text { infections }\end{array}$ & NA. Wound surveillance \\
\hline
\end{tabular}

${ }^{*} \mathrm{DB}=$ double blind; patient and carer were blinded to treatment; $\mathrm{NA}=$ not assessed.

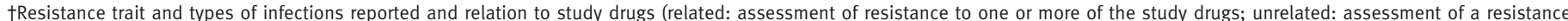
trait unrelated to the study antibiotics) and surveillance cultures reported. 


\begin{tabular}{|c|c|c|c|c|c|c|}
\hline Study & $\begin{array}{l}\text { Antibiotic } \\
\text { prophylaxis }\end{array}$ & Control & $\begin{array}{r}\text { Relati } \\
\left(95^{\circ}\right.\end{array}$ & $\begin{array}{l}\text { ive risk } \\
\% \mathrm{Cl})\end{array}$ & $\begin{array}{c}\text { Weight } \\
\text { (\%) }\end{array}$ & $\begin{array}{c}\text { Relative risk } \\
(95 \% \mathrm{Cl})\end{array}$ \\
\hline \multicolumn{7}{|c|}{ Systemic antibiotic prophylaxis (general) } \\
\hline Munster $1989^{59}$ & $1 / 22$ & $4 / 23$ & & & 10.8 & $0.26(0.03$ to 2.16$)$ \\
\hline Durtschi $1982^{56}$ & $1 / 25$ & $4 / 26$ & & & 10.8 & 0.26 (0.03 to 2.17$)$ \\
\hline Deutsch $1990^{55}$ & $8 / 15$ & $6 / 14$ & & & 17.1 & 1.24 (0.58 to 2.68$)$ \\
\hline Kimura $1998^{57}$ & $4 / 21$ & $7 / 19$ & $\rightarrow$ & & 20.3 & $0.52(0.18$ to 1.49$)$ \\
\hline De la Cal $2005^{53}$ & $6 / 53$ & $15 / 54$ & $\rightarrow$ & & 41.0 & 0.41 (0.17 to 0.97$)$ \\
\hline Total events & $20 / 136$ & $36 / 136$ & $\bullet$ & & 100.0 & $0.54(0.34$ to 0.87$)$ \\
\hline \multicolumn{7}{|c|}{ Test for heterogeneity: $\chi^{2}=5.85, \mathrm{df}=4, \mathrm{P}=0.21, \mathrm{I}^{2}=32 \%$} \\
\hline \multicolumn{7}{|c|}{ Test for overall effect: $z=2.51, P=0.01$} \\
\hline \multicolumn{7}{|c|}{ Systemic antibiotic prophylaxis (perioperative) } \\
\hline Steer $1997^{63}$ & $1 / 110$ & $2 / 110$ & - & & 27.9 & $0.50(0.05$ to 5.43$)$ \\
\hline Munster $1989^{59}$ & $1 / 6$ & $3 / 11$ & . & & 29.6 & 0.61 (0.08 to 4.67$)$ \\
\hline Alexander $1984^{50}$ & $5 / 35$ & $3 / 34$ & - & & 42.5 & $1.62(0.42$ to 6.25$)$ \\
\hline Total events & $7 / 151$ & $8 / 155$ & & & 100 & $1.01(0.38$ to 2.70$)$ \\
\hline \multicolumn{7}{|c|}{ Test for heterogeneity: $\chi^{2}=1.04, \mathrm{df}=2, \mathrm{P}=0.60, \mathrm{I}^{2}=0 \%$} \\
\hline \multicolumn{7}{|c|}{ Test for overall effect: $z=0.02, P=0.99$} \\
\hline \multicolumn{7}{|c|}{ Non-absorbable antibiotic prophylaxis } \\
\hline Barret $2001^{52}$ & 2/11 & $1 / 12$ & & & 100.0 & 2.18 (0.23 to 20.84 \\
\hline \multicolumn{7}{|c|}{ Test for heterogeneity: NA } \\
\hline \multicolumn{7}{|c|}{ Test for overall effect: $z=0.68, P=0.50$} \\
\hline \multicolumn{7}{|c|}{ Local antibiotic prophylaxis } \\
\hline Levine $1978^{65}$ & $6 / 12$ & $12 / 18$ & & 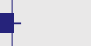 & 100.0 & $0.75(0.39$ to 1.44$)$ \\
\hline Test for heterogene & & & 0.010 .1 & 10 & 100 & \\
\hline \multicolumn{3}{|c|}{ Test for overall effect: $z=0.86, P=0.39$} & $\begin{array}{l}\text { Favours } \\
\text { prophylaxis }\end{array}$ & & & \\
\hline
\end{tabular}

Fig 3 | All cause mortality in burns patients according to type of antibiotic prophylaxis

erogeneity $\left(\mathrm{P}=0.21, \mathrm{I}^{2}=32 \%\right)$. The corresponding number needed to treat was 8 ( 5 to 33), with a control event rate of $26 \%$. The antibiotics used included cefotaxime, trimethoprim-sulfamethoxazole, penicillin, polymyxin $\mathrm{B}$, and a combination of oral neomycin, erythromycin, and nystatin. The exclusion of trials with inadequate allocation concealment increased benefit (0.42, 0.22 to 0.79 , three trials). There were no significant differences in mortality for perioperative nonabsorbable or topical antibiotic prophylaxis.

\section{Secondary outcomes}

Outcome definitions varied between the trials; table 4 summarises the results. Seven trials comprising 4835 patient days reported on bacteraemia. One trial administering perioperative teicoplanin prophylaxis showed a highly significant reduction (rate ratio $0.26,0.15$ to $0.45),{ }^{63}$ while all other trials, including those of the general setting, showed no significant differences, both individually and pooled. Five trials reported on pneumonia (mainly ventilator associated) (103 events, 2624 patient days). Use of systemic antibiotics in the general or perioperative setting showed a significant reduction in pneumonia (0.55 (0.36 to 0.84), three trials). Eleven trials reported on burn wound infection (not colonisation) (295 events, 7357 patient days). Perioperative systemic antibiotic prophylaxis had an advantage of borderline significance (0.72 (0.52 to 1.01$)$, four trials), while general systemic and topical antibiotics had no effect. Most trials did not report on length of admission to hospital in a manner that could be pooled.

Microbiological assessment showed that infection or colonisation by Paeruginosa was not significantly different, both in trials assessing antibiotics with and without an anti-pseudomonal spectrum of coverage (1.06, 0.66 to 1.71 , four trials; and $0.89,0.62$ to 1.28 , seven trials, respectively). $S$ aureus infections significantly decreased with anti-staphylococcal prophylaxis $(0.58$, 0.43 to 0.76 , six trials), while the three trials assessing an antibiotic without anti-staphylococcal coverage showed an overall increase but with significant heterogeneity $\left(\mathrm{I}^{2}=51 \%\right)$. Similarly, MRSA infections significantly decreased when anti-MRSA prophylaxis was used $(0.36,0.19$ to 0.70 , three trials). Fungal infections were documented more often with antibiotic prophylaxis, but without a significant difference $(1.58,0.63$ to 3.99, seven trials).

While most trials performed routine surveillance cultures, at least of burn wounds (table 2), results pertaining to induction of resistance were scarce (table 3). Isolation of bacteria resistant to the study antibiotics from any site was significantly higher in the intervention $\operatorname{arm}(2.84,1.38$ to 5.83 , three trials, one topical, two systemic). Three trials reported on resistant infections unrelated to the intervention antibiotic (MRSA or resistance to gentamicin), which was lower in the intervention $\operatorname{arm}(0.42,0.18$ to 0.98$)$.

Six trials addressed adverse events comparatively, of which three reported no events. Adverse events requiring discontinuation of antibiotic treatment were specified; these consisted of rash in two trials ${ }^{5163}$ and diarrhoea in one. ${ }^{55}$ There was higher rate of discontinuation because of adverse events with treatment overall $(4.97,1.08$ to 22.96$)$. Pseudomembraneous colitis was not reported.

\section{DISCUSSION}

The pooled evidence in our systematic review shows a significant decrease in all cause mortality with systemic antibiotic prophylaxis for 4-14 days among patients with burns (mostly severe), with a number needed to treat of 8 (5 to 33). Systemic prophylaxis was associated with a reduced rate of pneumonia and, when administered perioperatively, with a reduced rate of burn wound infections. Resistance of bacteria to the antibiotic used for prophylaxis increased. Our findings are based on a few small trials and in most randomisation methods were unclear or clearly inadequate. These results stand in contrast with the current consensus regarding antibiotic prophylaxis for patients with severe burns. ${ }^{14-18}$

Comparison with studies conducted in intensive care units More evidence on the effects of antibiotic prophylaxis is available from studies on other critically ill patients in intensive care units. In this setting prophylaxis with non-absorbable or topical (oropharyngeal) antibiotics aims to decontaminate the digestive tract of Gram negative bacteria, $S$ aureus, and candida. Most trials assessing antibiotic prophylaxis in intensive care 
Table 4 |Secondary outcomes in burns patients according to antibiotic treatment

\begin{tabular}{|c|c|c|c|c|}
\hline \multirow[b]{2}{*}{ Outcome } & \multirow[b]{2}{*}{ No of trials } & \multirow[b]{2}{*}{ Rate ratio $(95 \% \mathrm{Cl})$} & \multicolumn{2}{|c|}{ Heterogeneity } \\
\hline & & & $X^{2} P$ value & 12 \\
\hline \multicolumn{5}{|l|}{ Bacteraemia* } \\
\hline Systemic general & $4^{53555662}$ & $1.30(0.91$ to 1.85$)$ & 0.56 & $0 \%$ \\
\hline Topical, inhalation & $1^{65}$ & $0.92(0.39$ to 2.16$)$ & - & - \\
\hline \multicolumn{5}{|l|}{ Wound infection } \\
\hline Systemic general & $5^{5355566264}$ & $1.13(0.82$ to 1.55$)$ & 0.92 & $0 \%$ \\
\hline Systemic perioperative & $4^{50516163}$ & $0.72(0.52$ to 1.01$)$ & 0.17 & $40 \%$ \\
\hline Topical & $2^{2154}$ & $1.49(0.67$ to 3.34$)$ & 0.83 & $0 \%$ \\
\hline \multicolumn{5}{|l|}{ Pneumonia } \\
\hline Total systemic & 3 & $0.55(0.36$ to 0.84$)$ & 0.28 & $21 \%$ \\
\hline Systemic general & $2^{5357}$ & $0.52(0.33$ to 0.83$)$ & 0.12 & $58 \%$ \\
\hline Systemic perioperative & $1^{63}$ & $0.71(0.23$ to 2.23$)$ & - & - \\
\hline Non-absorbable & $1^{52}$ & $2.70(0.11$ to 66.10$)$ & - & - \\
\hline Topical, inhalation & $1^{65}$ & $1.00(0.42$ to 2.37$)$ & - & - \\
\hline \multicolumn{5}{|l|}{$P$ aeruginosa infections } \\
\hline Total & 12 & $0.95(0.71$ to 1.27$)$ & 0.94 & $0 \%$ \\
\hline With anti-pseudomonal activity & $4^{55586465}$ & $1.06(0.66$ to 1.71$)$ & 0.97 & $0 \%$ \\
\hline Without anti-pseudomonal activity & $7^{5153565762-64}$ & $0.89(0.62$ to 1.28$)$ & 0.61 & $0 \%$ \\
\hline \multicolumn{5}{|l|}{ S aureus infections } \\
\hline With anti-staphylococcal activity & $6^{51575862-64}$ & $0.58(0.43$ to 0.76$)$ & 0.72 & $0 \%$ \\
\hline Without anti-staphylococcal activity & $3^{535556}$ & 1.70 (1.09 to 2.64$)$ & 0.13 & $51 \%$ \\
\hline \multicolumn{5}{|l|}{ Fungal infection } \\
\hline Total & 7 & 1.58 (0.63 to 3.99$)$ & 0.56 & $0 \%$ \\
\hline Antibacterials and antifungals & $3^{525355}$ & $1.26(0.26$ to 6.14$)$ & 0.49 & $0 \%$ \\
\hline Antibacterials without antifungals & $4^{21566263}$ & $1.78(0.56$ to 5.59$)$ & 0.34 & $11 \%$ \\
\hline \multicolumn{5}{|l|}{ Resistance development† } \\
\hline Resistance trait related to prophylaxis & $3^{535463}$ & 2.15 (1.25 to 3.70$)$ & 0.50 & $0 \%$ \\
\hline Resistance trait unrelated to prophylaxis & $3^{215658}$ & $0.42(0.18$ to 0.98$)$ & 0.70 & $0 \%$ \\
\hline \multicolumn{5}{|l|}{ Adverse events requiring discontinuation $†$} \\
\hline Total & 3 & 4.97 (1.08 to 22.96$)$ & 0.41 & $0 \%$ \\
\hline Systemic general & $1^{55}$ & $13.10(0.65$ to 265.42$)$ & - & - \\
\hline Systemic perioperative & $2^{5163}$ & 2.99 (0.47 to 19.02$)$ & 0.32 & 0 \\
\hline \multicolumn{5}{|c|}{$\begin{array}{l}\text { *With systemic prophylaxis in perioperative setting, one trial }{ }^{63} \text { showed highly significant advantage with prophylaxis }(0.26,0.15 \text { to } 0.45) \text {, while } \\
\text { other }{ }^{50} \text { showed no difference }(1.16,0.79 \text { to } 1.70) \text {, thus this category was not pooled and nor was overall assessment of systemic antibiotic } \\
\text { prophylaxis. } \\
\text { †Risk ratios shown. }\end{array}$} \\
\hline
\end{tabular}

units, however, also used broad spectrum systemic antibiotics for the first few days. The full (systemic plus non-absorbable) selective digestive decontamination regimen achieves a larger reduction in mortality (odds ratio $0.71,0.61$ to 0.82 ) than the non-absorbable intervention alone $(0.94,0.71$ to 1.24$) .{ }^{1169}$ Selective decontamination regimens reduce mainly Gram negative infections, ${ }^{70}$ and induction of resistance has not been shown in trials conducted in low resistance settings. ${ }^{12}$ In the trials that assessed burns patients, systemic antibiotics alone were used in all the perioperative trials and some of the general prophylaxis trials. A recent trial, independently showing a reduction in mortality and ventilator associated pneumonia, used the full selective decontamination regimen. ${ }^{53} S$ aureus infections were reduced with prophylaxis in the perioperative setting. Considering similar risk factors for intensive care and burns patients, the unique susceptibility of burns patients to infections caused by skin flora, and the available evidence, it seems that the optimal regimen for prophylaxis among burns patients would be a full selective decontamination regimen including systemic and non-absorbable antibiotics. Antibiotics targeting Gram positive bacteria might be of added value perioperatively after discontinuation of the systemic antibiotic.

\section{Strengths and limitations of study}

We included systemic, non-absorbable, and topical antibiotics to inspect the effects of each separately and to fully appraise their combined effect on resistance induction. We included all types of burns, although the question of prophylaxis applies mainly to patients with severe burns. Most trials recruited patients with burns over more than $20 \%$ of total body surface area, and the mortality rate of the control group was $25 \%$ in trials that assessed general systemic prophylaxis and $17 \%$ in all trials reporting on mortality (fig 3). The paucity of trials precluded separate analyses for patients with severe or full thickness burns only. 


\section{WHAT IS ALREADY KNOWN ON THIS TOPIC}

Antibiotic prophylaxis reduces all cause mortality among patients in intensive care

Current guidelines for management do not recommend systemic antibiotic prophylaxis for burns patients, stating lack of evidence for efficacy and induction of antibiotic resistance

\section{WHAT THIS STUDY ADDS}

In burns patients systemic antibiotic prophylaxis administered in the first 4-14 days significantly reduces all cause mortality by nearly a half; limited perioperative prophylaxis reduces wound infections but not mortality

Topical antibiotic prophylaxis applied to burn wounds, commonly recommended, had no beneficial effects

The methodological quality of the evidence is weak, however, so a large, robust randomised controlled trial is now needed

\section{Implications for further research}

Future trials should assess a full selective decontamination regimen including systemic and non-absorbable antibiotics. The duration of the systemic component can probably be limited to the first four days, similar to the regimen used in the most recent trial and in trials in the intensive care unit. ${ }^{125377}$ Limited perioperative prophylaxis targeting Gram positive bacteria can be considered. Optimal resuscitation protocols and local care should be provided uniformly to both arms to assess the added benefit of antibiotic prophylaxis to current best practice. ${ }^{14-18}$ Special attention should be drawn to infection control practices during the trial to avoid cross infection between the trial arms. Contemporary methods used in multicentre trials should ensure adequate sequence generation and allocation concealment. Although randomised controlled trials might not be the optimal platform to assess development of resistance (randomised patients are in the same unit and the timeframe is inadequate), ${ }^{78}$ special attempts should be placed on documenting the effect of prophylaxis on colonisation (using surveillance cultures) and clinical infections caused by multidrug resistant bacteria. Other adverse effects including $C$ difficile colitis and fungal infections should be addressed. Ultimately, however, a patient's survival incorporates both ill effects and the benefit of prophylaxis and is the goal of managing burns patients. The current analysis $(26 \%$ mortality in the control arm and relative risk of 0.54) suggests that an individual multicentre trial can be powered to assess all cause mortality as the primary outcome (about 200 patients per arm for a power of $80 \%)$. In hospital mortality among burns patients is highly variable; a fixed point in time relevant to the assessment of benefit and harm should be used.

In summary, we have shown a discrepancy between current guidelines for management of burns patients recommending against antibiotic prophylaxis and the evidence showing a reduction of about $50 \%$ in all cause mortality with systemic antibiotic prophylaxis. Given the paucity and limitations of the available evidence, this should serve mainly as an urgent call for a large randomised controlled trial.

Contributors: MP was responsible for conception of the trial and is guarantor. TA, AL, and MP wrote the protocol, carried out searches, extracted and analysed the data, and wrote the manuscript. All authors critically revised the manuscript.

Funding: This research received no specific grant from any funding agency in the public, commercial, or not-for-profit sectors.

Competing interests: None declared.

Ethical approval: Not required

Data sharing: Analyses in RevMan software are available from the corresponding author at paulm@post.tau.ac.il.

1 Enoch S, Roshan A, Shah M. Emergency and early management of burns and scalds. BMJ 2009;338:b1037.

2 Sanghavi P, Bhalla K, Das V. Fire-related deaths in India in 2001: a retrospective analysis of data. Lancet 2009;373:1282-8.

3 Chim H, Tan BH, Song C. Five-year review of infections in a burn intensive care unit: high incidence of Acinetobacter baumannii in a tropical climate. Burns 2007;33:1008-14.

4 Wibbenmeyer L, Danks R, Faucher L, Amelon M, Latenser B, Kealey GP, et al. Prospective analysis of nosocomial infection rates, antibiotic use, and patterns of resistance in a burn population. J Burn Care Res 2006;27:152-60. 
5 Fitzwater J, Purdue GF, Hunt JL, O’Keefe GE. The risk factors and time course of sepsis and organ dysfunction after burn trauma. J Trauma 2003;54:959-66.

6 Sharma BR. Infection in patients with severe burns: causes and prevention thereof. Infect Dis Clin North Am 2007;21:745-59,ix.

7 Herndon DN, Lal S. Is bacterial translocation a clinically relevant phenomenon in burns? Crit Care Med 2000;28:1682-3.

8 Gamelli RL, He LK, Liu LH. Macrophage mediated suppression of granulocyte and macrophage growth after burn wound infection reversal by means of anti-PGE2. J Burn Care Rehabil 2000;21:64-9.

9 Hunt JP, Hunter CT, Brownstein MR, Giannopoulos A, Hultman CS, de Serres S, et al. The effector component of the cytotoxic T-lymphocyte response has a biphasic pattern after burn injury. J Surg Res 1998;80:243-51.

10 Shoup M, Weisenberger JM, Wang JL, Pyle JM, Gamelli RL, Shankar R. Mechanisms of neutropenia involving myeloid maturation arrest in burn sepsis. Ann Surg 1998;228:112-22.

11 Silvestri L, van Saene HK, Milanese M, Gregori D, Gullo A. Selective decontamination of the digestive tract reduces bacterial bloodstream infection and mortality in critically ill patients. Systematic review of randomized, controlled trials. J Hosp Infect 2007;65:187-203.

12 De Smet AM, Kluytmans JA, Cooper BS, Mascini EM, Benus RF, van der Werf TS, et al. Decontamination of the digestive tract and oropharynx in ICU patients. N Engl J Med 2009;360:20-31.

13 Evidence-Based Guidelines Group; American Burn Association. Practice guidelines for burn care. J Burn Care Rehabil 2001;suppl:1 $67 \mathrm{~s}$.

14 Rice PL. Emergency care of moderate and severe thermal burns in adults. In: Marx JA, Grayzel J, eds. 2008. www.uptodate.com/ patients/content/topic.do?topicKey= Ay/xsdTHiuaQHr.

15 White CE, Renz EM. Advances in surgical care: management of severe burn injury. Crit Care Med 2008;36:S318-24.

16 D'Avignon LC, Saffle JR, Chung KK, Cancio LC. Prevention and management of infections associated with burns in the combat casualty. J Trauma 2008;64:S277-86.

17 Church D, Elsayed S, Reid O, Winston B, Lindsay R. Burn wound infections. Clin Microbiol Rev 2006;19:403-34.

18 Silver GM, Klein MB, Herndon DN, Gamelli RL, Gibran NS, Altstein L, et al. Standard operating procedures for the clinical management of patients enrolled in a prospective study of inflammation and the host response to thermal injury. J Burn Care Res 2007;28:222-30.

19 Ressner RA, Murray CK, Griffith ME, Rasnake MS, Hospenthal DR, Wolf SE. Outcomes of bacteremia in burn patients involved in combat operations overseas. J Am Coll Surg 2008;206:439-44.

20 Higgins JPT, Green S, eds. Cochrane handbook for systematic reviews of interventions: version 5.0.1 Cochrane Collaboration, 2008

21 Livingston DH, Cryer HG, Miller FB, Malangoni MA, Polk HC Jr, Weiner LJ. A randomized prospective study of topical antimicrobial agents on skin grafts after thermal injury. Plast Reconstr Surg 1990;86:1059-65.

22 Greminger RF, Elliott RA Jr, Rapperport A. Antibiotic iontophoresis for the management of burned ear chondritis. Plast Reconstr Surg 1980;66:356-60.

23 Jarrett F, Balish E, Moylan JA, Ellerbe S. Clinical experience with prophylactic antibiotic bowel suppression in burn patients. Surgery 1978;83:523-7.

24 Jarrett F, Chan CK, Balish E, Moylan J. Antibiotic bowel preparation and burn wound colonization. Surg Forum 1976;27:67-8.

25 LaForest NT, Cofrancesco C. Antibiotic iontophoresis in the treatment of ear chondritis. Phys Ther 1978;58:32-4.

26 Manson WL, Westerveld AW, Klasen HJ, Sauer EW. Selective intestinal decontamination of the digestive tract for infection prophylaxis in severely burned patients. Scand J Plast Reconstr Surg Hand Surg 1987;21:269-72.

27 Rizzo A. [Use of cortisone-antibiotic ointment in the treatment of burns in pediatric surgery]. Minerva Chir 1974;29:463-9.

28 Kuzin MI, Kolker, II, Vares A, Fillippovich lu V, Makarenkova RV. [Antibacterial effect of preparations for the local treatment of burns and suppurative wounds]. Khirurgiia (Mosk) 1987:14-8.

29 Desai MH, Rutan RL, Heggers JP, Herndon DN. Candida infection with and without nystatin prophylaxis. An 11-year experience with patients with burn injury. Arch Surg 1992;127:159-62.

30 lakovlev VP, Krutikov MG, Alekseev AA, Grishina IA, Izotova GN, Kashin lu D. [A trial of using sulperazone (cefoperazone/sulbactam) in the combined treatment of patients with a burn infection]. Antibio Khimioter 1995;40:38-41.

31 Krutikov MG. [A trial of the clinical use of Cifran in treating infections in burn wounds and the infectious complications of burns]. Antibio Khimioter 2000;45:37-40.

32 Xu W, Deng S, Han C, Li X, Liao Z, Wang W. [A multicenter clinical trial of piperacillin/tazobactam in burn infection]. Zhonghua Shao Shang Za Zhi 2002;18:75-7.

33 lakovlev VP, Blatun LA, Krutikov MG, Puchkova LS, Izotova GN, Svetukhin AM, et al. [Use of cefpirome in the treatment of patients with skin and soft tissue infections]. Antibiot Khimioter 1996;41:24-9.

34 Barret JP, Dziewulski P, Ramzy PI, Wolf SE, Desai MH, Herndon DN Biobrane versus $1 \%$ silver sulfadiazine in second-degree pediatric burns. Plast Reconstr Surg 2000;105:62-5.

35 Donati L, Periti P. Antibiotic treatment of burned patients: an Italian multicentre study. Intensive Care Med 1994;20 Suppl 4:S30-4.

36 Donati L, Periti P, Andreassi A, Dioguardi D, Gliori A, Landi G, et al. Increased burn patient survival with once-a-day high dose teicoplanin and netilmicin. An Italian multicenter study. J Chemother 1998;10:47-57.

37 Lawrence JC, Cason JS, Kidson A. Evaluation of phenoxetolchlorhexidine cream as a prophylactic antibacterial agent in burns. Lancet 1982;i:1037-40.

38 Lowbury EJ, Lilly HA, Cason JS, Jackson DM, Bull JP, Davies JW, et al. Alternative forms of local treatment for burns. Lancet 1971;ii:110511.

39 Demling RH, DeSanti L. Management of partial thickness facial burns (comparison of topical antibiotics and bio-engineered skin substitutes). Burns 1999;25:256-61.

40 Sinha R, Agarwal RK, Agarwal M. Povidone iodine plus neosporin in superficial burns-a continuing study. Burns 1997;23:626-8.

41 Soroff HS, Sasvary DH. Collagenase ointment and polymyxin B sulfate/bacitracin spray versus silver sulfadiazine cream in partialthickness burns: a pilot study. J Burn Care Rehabil 1994;15:13-7.

42 Miller LM, Carroll WB, Hansbrough JF. The effect of antimicrobial prophylaxis for burn wound excision: ceforanide versus cefazolin. Curr Ther Res 1987;41:946-51.

43 Proctor DS. The treatment of burns: a comparative trial of antibiotic dressings. S Afr Med J 1971;45:231-6.

44 Steer JA, Papini RP, Wilson AP, McGrouther DA, Parkhouse N. Teicoplanin versus flucloxacillin in the treatment of infection following burns. J Antimicrob Chemother 1997;39:383-92.

45 Kunst MW. Ceftazidime treatment in severe burns. Serious infections in patients with severe burns treated with ceftazidime. Scand J Plast Reconstr Surg Hand Surg 1987;21:281-2.

46 Periti P, Stringa G, Donati L, Mazzei T, Mini E, Novelli A. Teicoplaninits role as systemic therapy of burn infections and as prophylaxis for orthopaedic surgery. Italian Study Groups for Antimicrobial Prophylaxis in Orthopaedic Surgery and Burns. Eur J Surg Suppl 1992:567:3-8.

47 Theron EJ, Nel CJ. Treatment of septic burns with a third-generation cephalosporin (cefatriaxon). S Afr Med J 1983;64:816-7.

48 Culbertson GR, McManus AT, Conarro PA, McManus WF, Mason AD Jr, Pruitt BA Jr. Clinical trial of imipenem/cilastatin in severely burned and infected patients. Surg Gynecol Obstet 1987;165:25-8.

49 Alekseev AA, Krutikov MG, Smirnov SV, Malakhov SF, Filimonov AA. [Clinical results of Fortum and Zinacef trial in burn centers of Russia] Khirurgiia (Mosk) 1996:55-9.

50 Alexander JW, MacMillan, BG. Lack of beneficial effects of restricted prophylactic antibiotics for debridement and/or grafting of seriously burned patients. Bull Clin Rev Burn Injuries 1984;1:20.

51 Alexander JW, MacMillan BG, Law EJ, Krummel R. Prophylactic antibiotics as an adjunct for skin grafting in clean reconstructive surgery following burn injury. J Trauma 1982;22:687-90.

52 Barret JP, Jeschke MG, Herndon DN. Selective decontamination of the digestive tract in severely burned pediatric patients. Burns 2001;27:439-45.

53 De La Cal MA, Cerda E, Garcia-Hierro P, van Saene HK, Gomez-Santos D, Negro E, et al. Survival benefit in critically ill burned patients receiving selective decontamination of the digestive tract: a randomized, placebo-controlled, double-blind trial. Ann Surg 2005;241:424-30.

54 Desai MH, Rutan RL, Heggers JP, Alvarado MI, McElroy K, Henrdon DN. The role of gentamicin iontophoresis in the treatment of burned ears. J Burn Care Rehabil 1991:12:521-4.

55 Deutsch DH, Miller SF, Finley RK, Jr. The use of intestinal antibiotics to delay or prevent infections in patients with burns. J Burn Care Rehabil 1990;11:436-42.

56 Durtschi MB, Orgain C, Counts GW, Heimbach DM. A prospective study of prophylactic penicillin in acutely burned hospitalized patients. J Trauma 1982;22:11-4.

57 Kimura A, Mochizuki T, Nishizawa K, Mashiko K, Yamamoto Y, Otsuka T. Trimethoprim-sulfamethoxazole for the prevention of methicillin-resistant Staphylococcus aureus pneumonia in severely burned patients. / Trauma 1998;45:383-7.

58 Lowbury EJ, Jackson DM. Local chemoprophylaxis for burns with gentamicin and other agents. Lancet 1968;1:654-7.

59 Munster AM, Xiao GX, Guo Y, Wong LA, Winchurch RA. Control of endotoxemia in burn patients by use of polymyxin B. J Burn Care Rehabil 1989;10:327-30.

60 Piel P, Scarnati S, Goldfarb IW, Slater H. Antibiotic prophylaxis in patients undergoing burn wound excision. J Burn Care Rehabil 1985;6:422-4. 
61 Ramos G, Resta M, Machare Delgado E, Durlach R, Fernandez Canigia L, Benaim F. Systemic perioperative antibiotic prophylaxis may improve skin autograft survival in patients with acute burns. Burn Care Res 2008;29:917-23.

62 Rodgers GL, Fisher MC, Lo A, Cresswell A, Long SS. Study of antibiotic prophylaxis during burn wound debridement in children. J Burn Care Rehabil 1997;18:342-6.

63 Steer JA, Papini RP, Wilson AP, McGrouther DA, Nakhla LS, Parkhouse N. Randomized placebo-controlled trial of teicoplanin in the antibiotic prophylaxis of infection following manipulation of burn wounds. Br J Surg 1997;84:848-53.

64 Ugburo AO, Atoyebi OA, Oyeneyin JO, Sowemimo GO. An evaluation of the role of systemic antibiotic prophylaxis in the control of burn wound infection at the Lagos university teaching hospital. Burns 2004;30:43-8.

65 Levine BA, Petroff PA, Slade CL, Pruitt BA Jr. Prospective trials of dexamethasone and aerosolized gentamicin in the treatment of inhalation injury in the burned patient. J Trauma 1978;18:188-93.

66 Garner JS, Jarvis WR, Emori TG, Horan TC, Hughes JM. CDC definitions for nosocomial infections, 1988. Am J Infect Control 1988;16:128-40.

67 Peck MD, Weber J, McManus A, Sheridan R, Heimbach D. Surveillance of burn wound infections: a proposal for definitions. J Burn Care Rehabil 1998;19:386-9.

68 Pruitt BA, Goodwin CW, Pruitt SK, Cleon W. Burn, including cold, chemical and electric injuries. In: Sabiston DC, ed. Textbook of surgery. 15th ed. Saunders, 1997:221-52.

69 Silvestri L, van Saene HK, Weir I, Gullo A. Survival benefit of the full selective digestive decontamination regimen. J Crit Care 2009;24:474 e7-14.

70 Silvestri L, van Saene HK, Casarin A, Berlot G, Gullo A. Impact of selective decontamination of the digestive tract on carriage and infection due to Gram-negative and Gram-positive bacteria: a systematic review of randomised controlled trials. Anaesth Intensive Care 2008;36:324-38.

71 Greenhalgh DG, Saffle JR, Holmes JH 4th, Gamelli RL, Palmieri TL, Horton JW, et al. American Burn Association consensus conference to define sepsis and infection in burns. J Burn Care Res 2007;28:776-90.

72 Boyce JM, White RL, Causey WA, Lockwood WR. Burn units as a source of methicillin-resistant Staphylococcus aureus infections. JAMA 1983;249:2803-7.

73 Richard P, Le Floch R, Chamoux C, Pannier M, Espaze E, Richet H. Pseudomonas aeruginosa outbreak in a burn unit: role of antimicrobials in the emergence of multiply resistant strains. J Infect Dis 1994;170:377-83.

74 Simor AE, Lee M, Vearncombe M, Jones-Paul L, Barry C, Gomez M, et al. An outbreak due to multiresistant Acinetobacter baumannii in burn unit: risk factors for acquisition and management. Infect Control Hosp Epidemiol 2002;23:261-7.

75 Oliveira GA, Dell'Aquila AM, Masiero RL, Levy CE, Gomes MS, Cui L, et al. Isolation in Brazil of nosocomial Staphylococcus aureus with reduced susceptibility to vancomycin. Infect Control Hosp Epidemiol 2001;22:443-8.

76 Falk PS, Winnike J, Woodmansee C, Desai M, Mayhall CG. Outbreak of vancomycin-resistant enterococci in a burn unit. Infect Control Hosp Epidemiol 2000;21:575-82.

77 De Jonge E, Schultz MJ, Spanjaard L, Bossuyt PM, Vroom MB Dankert J, et al. Effects of selective decontamination of digestive tract on mortality and acquisition of resistant bacteria in intensive care: randomised controlled trial. Lancet 2003;362:1011-6.

78 Gafter-Gvili A, Paul M, Fraser A, Leibovici L. Effect of quinolone prophylaxis in afebrile neutropenic patients on microbial resistance: systematic review and meta-analysis. J Antimicrob Chemother 2007;59:5-22

Accepted: 24 November 2009 